\title{
Design of Structure and Pneumatic System of Parts Moving Manipulator Yu Wei
}

\author{
(NanChang Institute of Science \& Technology, Nanchang, 330108)
}

\section{Keywords: Economic; Manipulator; PLC; Structure design}

\begin{abstract}
Manipulator is a powerful tool which can help people to carry out the safe operation, production automation and improve the productivity of labor. Based on the summary of the situation of research and development of manipulator, this article analyzes the functions of parts moving manipulator and carries out mechatronic design of parts moving manipulator according to the practical project items of parts moving manipulator of enterprises. On the basis of the analysis of the performance requirement and the operating characteristics of parts moving manipulator, this article analyses and designs the whole schemes for the mechanical structure, driving system, driving mode and the software and hardware control system of manipulator, and in which, the form of mechanical structure of cylindrical coordinate system is determined to be adopted in the design of manipulator, the driving scheme of pneumatic transmission is adopted, and the system control is carried out by PLC. On this basis, this article analyses the kinematics and dynamics of parts moving manipulator and summarizes the relationship between displacement, speed, acceleration and joint angle.
\end{abstract}

\section{Introduction}

With the progress of science and technology and the development of social economy, the application area of manipulator has been becoming wider and wide. The manipulator can be found everywhere in human society. The application of manipulator has been extended to the civilian application fields such as architecture field, digging field and service and entertainment field from the high science fields such as ocean development and space exploration, and various manipulators are emerging and being applied in all fields at an alarming speed. Manipulator is the result of the development of production level of human and the improvement of the product quality and the production efficiency of products. Because manipulator is good at finishing the repeating, tedious work with high requirement of precision, and it can replace human to finish the works human can not finish or do not want to finish in abominable conditions. However, the emergence of manipulator greatly liberate the productive forces of human. So the development of manipulator is the result and the inexorable trend of the social development. Because of the rapid development of manipulator, it is inevitable that manipulator appears in the school teaching. As the typical product of manipulator, the design and application of the manipulator with four freedom of motion has very important significance for the professional teachings and researches of mechanotronics, mechanical structure technology, machine building, automation and electronic information.

\section{Basic Structure and Types of Manipulator}

Although the appearances and shapes of manipulators are very different from each other, and their functions are also different form each other. On the system of manipulator, the main components of manipulator are very similar which can be divided into the four parts the executing agency for the last execution, the power-driven system of manipulator, the control system used to control manipulator and the man-machine interface. The man-machine interface is the key part which can let the manipulator system finish work following one person's intention and carry out the information exchange between one person and the machine. The control precision and the reliability of manipulator are determined by the core part of manipulator - control system of manipulator. The forms of control system of manipulator can be generally divided into two types centralized control and distributed control. Centralized control structure refers to a control structure in which all 
information input and computing are centralized in a computer. Obviously, sample structure is one of the characteristics of centralized control structure, but it also need a computer with high performance.

For distributed control system, the effects to the whole system are smaller than the effects to the centralized control system when a fault of one subsystem occurs. According to different driven power source, manipulator also can be divided into three types pneumatic drive manipulator, electric drive manipulator and hydraulic drive manipulator. The advantages of pneumatic drive manipulator are convenient drive and safe operation, the disadvantages of pneumatic drive manipulator are great shock and difficult precision control. The characteristics of hydraulic drive manipulator are high power and smooth operation, but it is not secure. The advantages of electric drive manipulator are good performance and convenient control, but its price is very high. For round cylindrical coordinate type manipulator, it is not convenient to protect the prismatic joint that is the disadvantage of round cylindrical coordinate type manipulator, but compared with other manipulators, it has many advantages in space such as intuitive positioning, large movement range and small footprint. So it is widely used as teaching and experiment type manipulator.

\section{Coordinate Form and Degree of Freedom of Manipulator}

As the last executing agency of manipulator, the distribution and type of mechanical structure can directly affect the drive system and the transmission way of manipulator, even the working performance of the whole manipulator. Manipulator can be divided into many types such as rectangular coordinates, spherical coordinate, cylindrical coordinates, multi-joint type and other types according to the coordinate form of manipulator. Rectangular coordinates type manipulator has many advantages such as sample structure, convenient solving of space trajectory, high positioning precision, the joints without coupling, relatively independent movements of the manipulator's sticks, convenient movements and control and no singular states produced. At the same time, it also has some disadvantages such as big space needed, small range of executing movements and inflexible operation. The main characteristics of SCARA type manipulator are sample structure and rapid response to order which is often used in the assembling work of parts in the vertical direction. Joint type manipulator has the highest operation flexibility, but it is very hard to carry out good control because of the complex kinematic analysis.

For parts moving manipulator, the movement forms of the mechanical arm include lifting, lowering, extending, retracting and rotating when manipulator is moving parts. For meet the requirements of functions, there are four freedom degrees of the parts moving manipulator designed in this article. In addition, the movement form of manipulator is divided into two parts direction movement and work execution. Among which, the part of direction movement covers two freedom degrees which include extending and retracting movement agency and plane swing agency. Because there is no coupling between the two freedom degrees, the computing and control of manipulator are effectively simplified. The work execution part also has two freedom degrees, and the two freedom degrees are orthogonal which include lifting and lowering agency and clamping and relaxing agency which are shown as figure 1. 


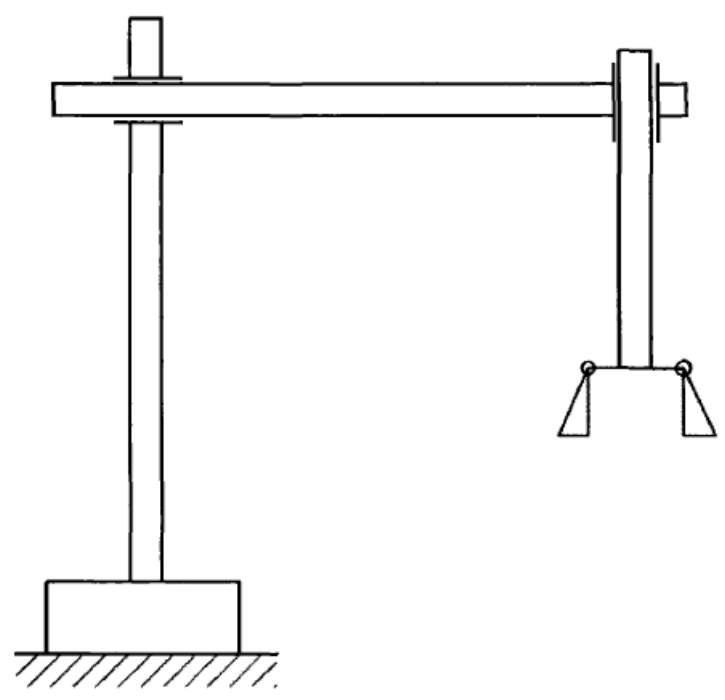

Figure 1 Structure Diagram of Manipulator

\section{Performance Analysis and Scheme Design of the Drive System of Manipulator}

Pneumatic drive, hydraulic drive and electric drive are the main three forms of the drive ways of manipulator at present. Considering that the parts moving manipulator is mainly used in teaching and experiment in which high requirement of precision is not needed, this article determine to adopt the pneumatic drive after the comparison of all kinds of drive forms.

The component pneumatic drive system of pneumatic system is a energy conversion system. The typical pneumatic transmission system consists of air source device, execute component, control element and auxiliary components shown as figure 2 .

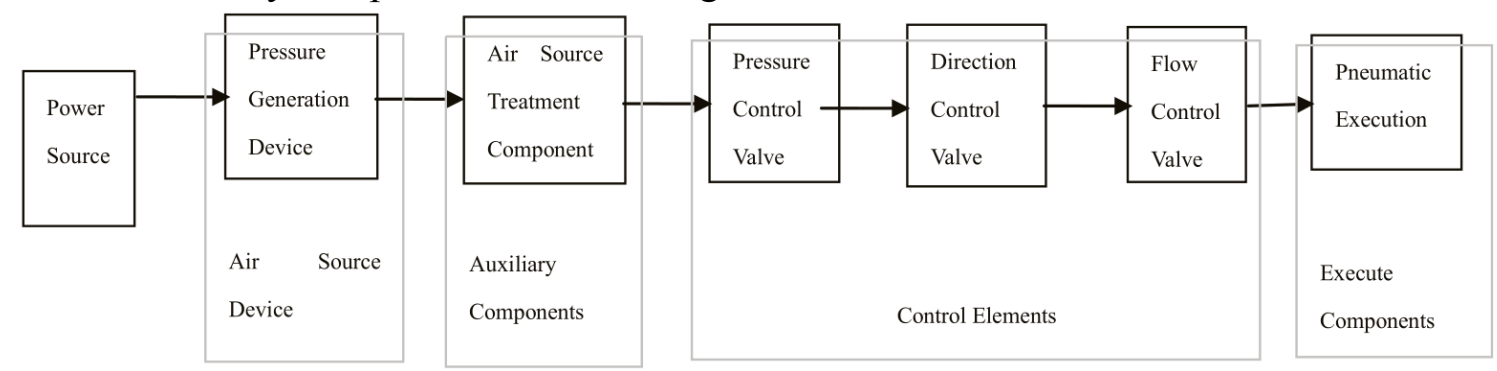

Figure 2 Basic Components of Pneumatic System

Pressure generation device is a energy device for air of which the shorthand is air source device, and its main part is air compressor, in addition, there is air source purification equipment.

Auxiliary components refer to the devices such as distributing water and trapping air equipment, air regulator, muffler and all kinds of pipe accessories which are used to make air purified, lubricated and anechoic and connect different components.

Control elements are also called operation, computing and testing elements which are used to control the pressure, the flow and flow direction of the compressed air in order to make the execute agency finish the scheduled moving rules. Control elements include various pressure valves, direction valves, flow valves, logic elements, fluidic elements, trip valves and sensors.

Execute components refer to an energy conversion devices which are used to transfer hydraulic power of compressed air to mechanical power such as air cylinder with left straight reciprocating motion, air motor with continuous rotary motion and rotary actuator without continuous rotary motion.

Air source device provides compressed air for pneumatic system with compressed air as the working substance. Its main part is air compressor, in addition, it also includes compressed air purifying device and transmission pipeline. Air is a mixture of gases, its major compositions are nitrogen and oxygen, and there are hydrogen, less carbon dioxide and other gases. 
Based on the requirement of quality of compressed air of pneumatic system, air source device must provides enough clean and dry compressed air with certain pressure and flow for the pneumatic system. Following are the requirements.

Compressed air should has certain pressure and enough flow.

Compressed air should has a certain degree of purification.

The pressure fluctuation of compressed air should not be big and can be kept within a certain range.

Although the compressed air produced by air compressor can meet the requirements of the working pressure and working flow of pneumatic system, it has very high temperature, and it contains many pollutants such as evaporated lubricating oil, water vapor and dust which will be harmful to pneumatic system.

Pneumatic drive system has some advantages such as easily available air source, small effects of external temperature to compressed air, low price and easy modularization. The disadvantages of pneumatic drive system are great noise of the pressure transmission and exhausting and small thrust. The former disadvantage can be improved with the help of noise absorbing materials and muffler, and the later disadvantage is not a real disadvantage, because pneumatic drive system with small thrust is suited for the manipulator used in teaching and experiment.

\section{Conclusion}

Manipulator is a powerful tool which can help people to carry out the safe operation, production automation and improve the productivity of labor. Based on the summary of the situations of research and development of manipulator in recent years, this article roundly analyzes the functions of parts moving manipulator, carries out mechatronic design of parts moving manipulator according to the practical project items of parts moving manipulator, analyzes the kinematics and dynamics of manipulator and researches the functions of control system. On the basis of the rounded analysis of the work requirements and the characteristics of movements of parts moving manipulator, this article carries out the software and hardware design for mechanical system and control system of parts moving manipulator according to the analytic results of kinematics and dynamics of parts moving manipulator, and parts moving manipulator with four freedom degrees is lastly developed applied to teaching experiment platform.

\section{Reference}

[1] Song S, Wang Z, Wu W. Course Teaching Reform of Machinery Manufacturing Equipment based on PETOE[J]. China Educational Technology \& Equipment, 2012.

[2] Mao R Z. New motion control technology in the automation of machinery industry[J]. World Nonferrous Metals, 2016.

[3] Yuling L I. Course Group Experimental Teaching of Machine Design Manufacture and Automation for Excellent Engineers Education Program[J]. Experiment Science \& Technology, 2014.

[4] Wang X, Ya-Ping F U. Applied Research for Detection Technology in Automated Machinery Manufacturing System[J]. Coal Mine Machinery, 2012.

[5] Wen Y F. Application of Machinery and Equipment Automation Technology[J]. Coal Technology, 2010.

[6] Wen gui L I, Tao G B, Yan X C, et al. The Specialized Courses System Reform and Practice of Machine Design Manufacture and Automation Speciality[J]. Journal of Chongqing University, 2003.

[7] Gao G B, Liu W Q, Xiao H X. Development of the Purchase Management System Based on Web for the Machinery Manufacturing SMEs ERP[J]. Machine Building \& Automation, 2007.

[8] Zhang C H, Ying-Qiang Y I, Zhou H Z. Discussion on the Automation Technology 's Application in Mechanical Manufacturing[J]. Equipment Manufacturing Technology, 2012.

[9] Guan T Y. On teaching reform of course of Machinery Manufacturing Technology 
Foundation[J]. Journal of Changchun Institute of Technology, 2010.

[10]Chen J Y. Machinery manufacturing process status and development trend[J]. Mechanical Research \& Application, 2011.

[11]Jain A. Virtual commissioning of modular automation systems[C]// Intelligent Manufacturing Systems. 2010:72-77.

[12]Jia Y, Fu Q, Ma D S, et al. Power Distribution Automation System in Green Power Engineering[J]. Applied Mechanics \& Materials, 2013, 340:1034-1038. 